\title{
Polarized Atomic Hydrogen Target at MESA
}

\author{
V. Tyukin* and K. Aulenbacher ${ }^{\dagger}$ \\ Inst. of Nucl. Phys., JGU Mainz, Germany \\ E-mail: tioukine@kph.uni-mainz.de
}

One aim for the new electron accelerator MESA is to measure the weak mixing angle in electron proton scattering with high precision. This results in a requirement for beam polarization measurement of $\Delta P / P \approx 0.5 \%$. The Møller polarimeter proposed by $\mathrm{V}$. Luppov and E. Chudakov opens the way to reach a sufficiently accurate measurement. The polarized atomic hydrogen target is under construction. The current status including recent modifications is presented.

The 18th International Workshop on Polarized Sources, Targets, and Polarimetry, PSTP2019

23-27 September, 2019

Knoxville, Tennessee

\footnotetext{
* Speaker.

${ }^{\dagger}$ This project was supported by Deutsche Forschungsgemeinschaft (DFG) through the cluster of excellence PRISMA $^{+}$and SFB 1044
} 


\section{Introduction}

\subsection{The P2-experiment}

The new $\mathrm{cw}$-accelerator MESA [1] is presently being build at the institute of nuclear physics in Mainz/Germany. Begin of operation for experiments is planned for 2023. In the first years of service about $1 / 3$ of the experiments will be done in the new energy-recovery mode for the MAGIX experiment whereas the remaining major part of beam time will be done in conventional external beam mode and serves the $\mathrm{P} 2$-experiment [2]. P2 aims at a precision measurement of the electroweak mixing angle, it measures the parity violating rate asymmetry in the elastic scattering of longitudinally polarized electrons off protons. This asymmetry is proportional to the beam polarization. Assuming a state of the art polarization of $85 \%$ [3], the asymmetry will be $40 \mathrm{ppb}$ only. The accuracy goal for the asymmetry is $1.6 \%$ relative, the largest contribution being statistics even after the envisaged data taking time of 10000 hours. Therefore, systematic errors must be suppressed significantly below one percent, in particular the accuracy of polarization measurement should be $\leq 0.5 \%$.

\subsection{The polarimeter chain at MESA}

Since the accuracy of the polarimetry is important for the interpretation of the P2-result it is a pressing demand to have independent beam polarization measurements which are based on different physical processes. The idea of the chain [4] is to have three polarimeters, each achieving high accuracy. The three polarimeters are positioned at different stages of MESA, as indicated in fig. 1. The first is based on double Mott-scattering [5] and has already been tested under the typical conditions expected at MESA [6]. The polarimeter operates at the kinetic energy of the electron source $(100 \mathrm{keV})$. The second polarimeter uses the $5 \mathrm{MeV}$ beam after the MESA preaccelerator and measures single scattering Mott-asymmetries. It will be similar to the one used at MAMI [7]. Both devices are destructive for the beam since they require to introduce solid targets. The third polarimeter in the chain is based on Møller scattering. This is a double polarization experiment requiring an electron spin polarized target. Chudakov and Luppov [8] have suggested to use completely electron-spin polarized hydrogen atoms instead of the commonly used ferromagnetic foils. This apparatus, called "Hydro-Møller" by us, is the subject of our paper. It would be placed in line with the P2-experiment (fig. 1) and allow measuring online. Laser-Compton backscattering would offer similar advantages but is difficult to use here because of the low beam energy at the P2-experiment. A discussion of the systematic uncertainties of the polarimeters mentioned in this section can be found in [9].

\subsection{Online requirement}

A series of long term observations of the scattering asymmetry obtained with the $3.5 \mathrm{MeV}$ polarimeter at MAMI during production runs for nuclear physics experiments is shown in figure 2. Due to the finite lifetime of the cathodes the quantum efficiency after 12 days has decreased. The cathode is then reactivated during the regular maintenance interval of MAMI. The measurements demonstrate a quite reproducible behavior after the different activations but a typical drift of polarization by $1 \%$ (relative) per day is observed. Regular monitoring of beam polarization several 


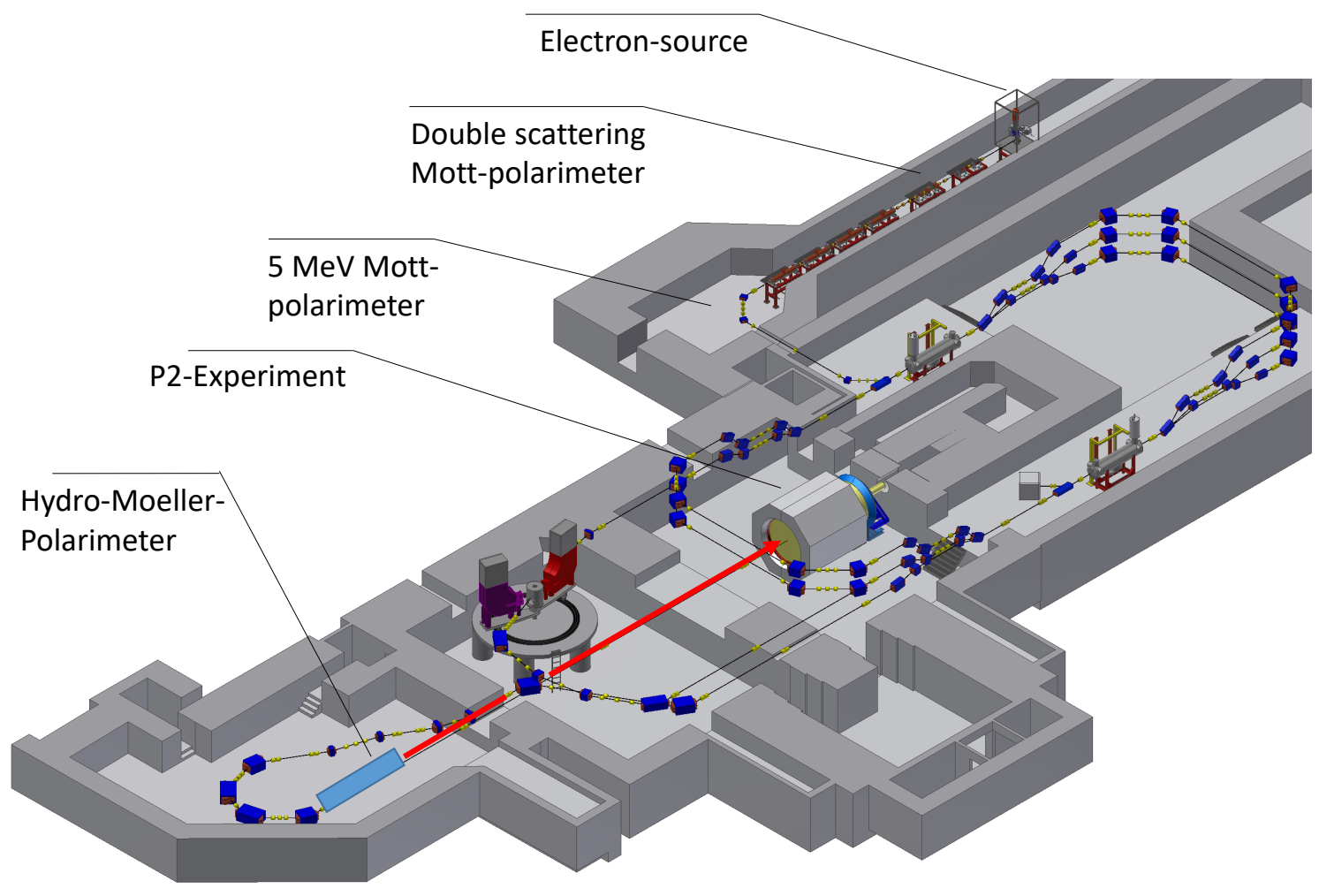

Figure 1: Mainz Energy-recovery Superconducting Accelerator (MESA). After leaving the source the beam passes a chain of three polarimeters. The final one, the Hydro-Møller-polarimeter, has online capability so that the beam can travel continuously from the polarimeter towards the P2-experiment (red arrow).

times a day is therefore needed in order to avoid additional errors due to interpolation of data intervals without polarization measurements. Therefore, an ideal polarimeter for P2 would operate online with low systematic error $(<0.5 \%$ relative) and sufficient statistical efficiency to provide a statistical error of about $0.25 \%$ after one day. This can in principle be achieved with the Hydro Møller.

\section{Concept of the Hydro-Møller polarimeter}

The basic concept proposed in [8] is illustrated in figure 2. A beam of unpolarized hydrogen atoms can be separated in the fringe field of a solenoid into two fractions. The fraction which is driven towards the homogeneous part of the solenoid and trapped there is almost completely electron-spin polarized if the magnetic field is much larger than the hyperfine splitting field. For $\mathrm{B}=8 \mathrm{~T}$ the polarization is $P=1-\varepsilon$ and $\varepsilon \approx 10^{-5}$. Compared to conventional double scattering experiments there is then no significant uncertainty caused by target polarization. Another important error source in Møller polarimeters is the enhancement of the spin polarized fraction of the target contributing to the detection process, the Levchuk effect [10]. It is also eliminated in this device since no further increase of polarization is possible. The hydrogen atoms can be stored with 


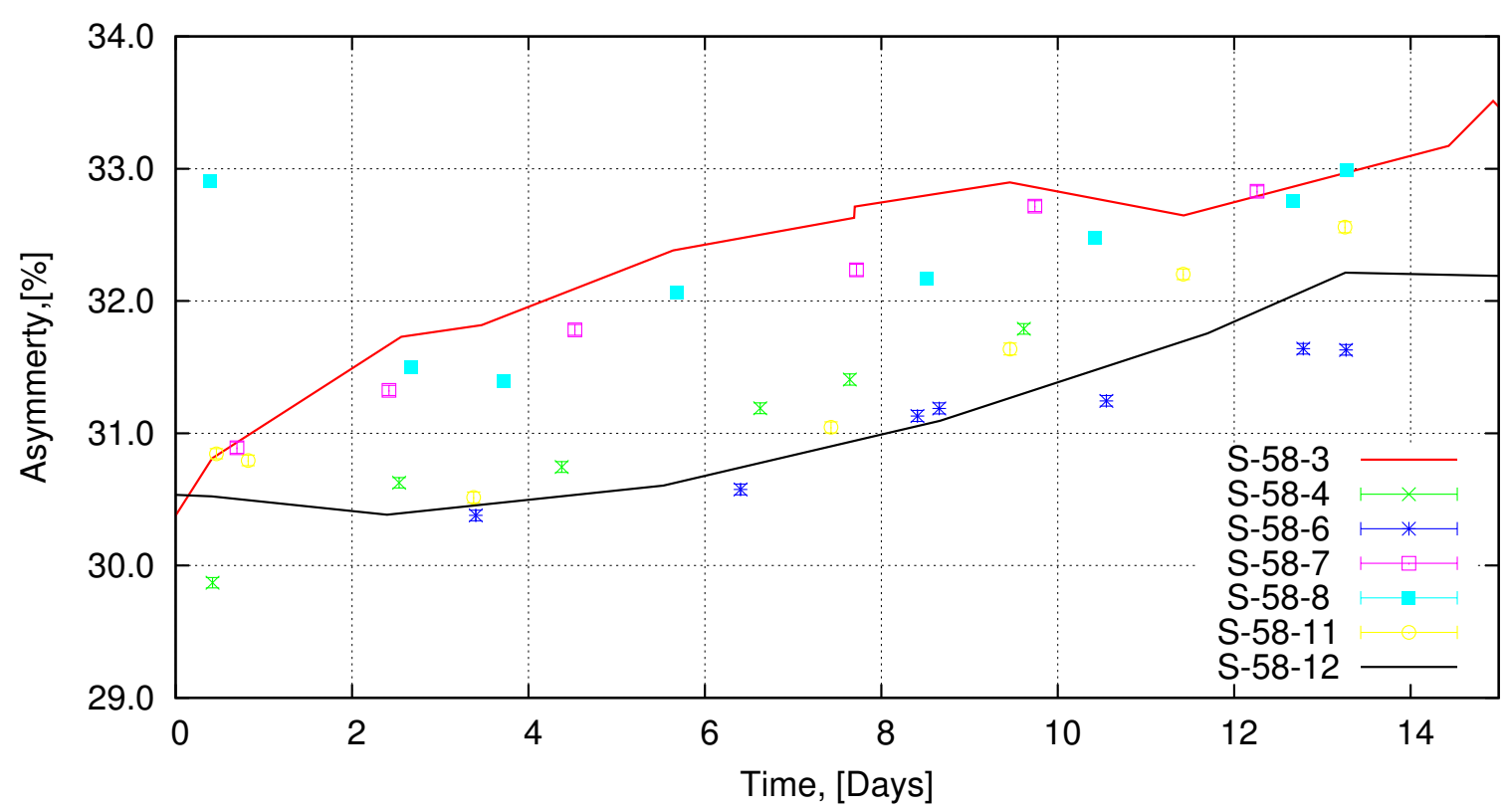

Figure 2: Measured Mott scattering asymmetry of superlattice cathode S-58 used at MAMI. Beam polarization can vary up to $10 \%$ (relative) during a run. Number from 01 to 12 stand for the different reactivations of the cathode surface. The first and last activation are indicated by red and black line respectively.

a particle density of $10^{15} \mathrm{~cm}^{-3}$. Assuming a target length of about $30 \mathrm{~cm}$, the areal density is of the order 100 nanogramms per $\mathrm{cm}^{2}$, so that the target is sufficiently transparent for the electron beam. On the other hand the count rate will be sufficient to meet the demand mentioned above. In [8] several practical aspects are discussed, in particular a potential dilution by neutral molecules and ions which all seem to be negligible. However, as simple as the concept may look like, it has not yet been realized in conjunction with a high power electron beam. A significant technological effort is necessary to realize such a concept, some of the difficulties will be illustrated in the next paragraphs.

\subsection{Polarized Hydrogen trap}

Hydrogen Atoms have to dissipate thermal energy inside the solenoid to become trapped. An obvious cooling mechanism is interaction with a cold wall which will on the other hand lead to rapid loss of atomic hydrogen due to recombination. It is possible to suppress this if the wall is covered with a film of suprafluid helium. In order to keep the Helium gas pressure low enough, the film must stay in a temperature range between 0.2 and $0.3 \mathrm{~K}$. A more detailed description of this type of trap and the achievable densities can be found in [11].

\subsection{Refrigerator}

A relatively large amount of heat will impinge on the cold surfaces of the trap, the main sources are thermal radiation, for instance through the beam pipe aperture, heat generated by hydrogen molecule formation and conduction. For the densities foreseen here the losses add up to $45 \mathrm{~mW}$. At temperatures $<0.3 \mathrm{~K}$ this requires a powerful ${ }^{3} \mathrm{He} /{ }^{4} \mathrm{He}$ dilution refrigerator with a ${ }^{3} \mathrm{He}$-flow of more than $40 \mathrm{mmol} / \mathrm{s}$. 


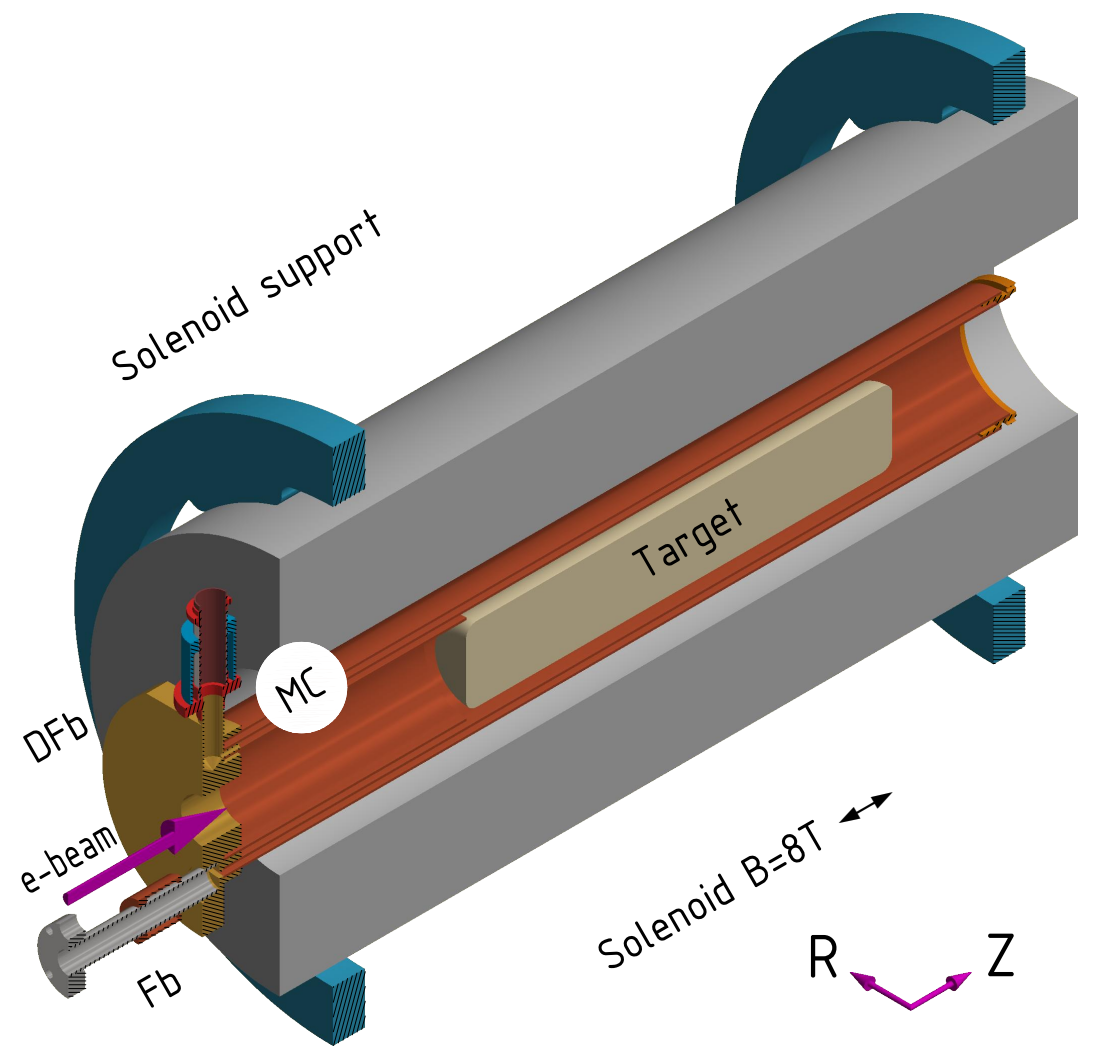

Figure 3: Schematic view of a Hydro-Møller target. The electron beam and hydrogen atoms enter at the left side of the solenoid. The inner side of the mixing chamber (MC) is covered with suprafluid ${ }^{4} \mathrm{He}$. FB and DFB film burners prevent ${ }^{4} \mathrm{He}$ losses from MC wall.

\subsection{Basic design of trap and refrigerator}

The refrigerator has to be compatible with several others devices, in particular the electron beam tube, the solenoid and a possibility to inject hydrogen atoms into the fringe field of the solenoid. Figure 5 presents a flow chart of the refrigerator. It contains two separate circuits ( ${ }^{4} \mathrm{He}$ precooling and the ${ }^{3} \mathrm{He} /{ }^{4} \mathrm{He}$ mixing circuit) which thermally interact through a series of heat exchangers. The mixing chamber where the lowest temperature is reached is essentially a double walled cylinder the inner surface of which is pointing towards the beam vacuum and supports the suprafluid film. Figure 4 shows a cross section of the basic design. The design has to meet several technical challenges. For some of them solutions have already been found whereas others still need engineering effort or even R\&D.

- The polarimeter has to run continuously over very long periods of time. Maintenance periods must be kept to minimum. We therefore have tried to minimize risks for cold leaks by choosing welded junctions where ever possible.

- ${ }^{4} \mathrm{He}$ precooling stage, achieving about $1 \mathrm{~K}$, is incorporated and will reduce liquid helium consumption below $101 / \mathrm{h}$. 
- Evaporation from the helium film has to be minimized by adequate "film-burners". The final design of these parts is not decided yet.

- Freezing out of the "wrong" hydrogen spin-state in the injection system may lead to clogging and can interrupt the continuous operation of the polarimeter. We are presently discussing two possibilities to handle this issue, see below.

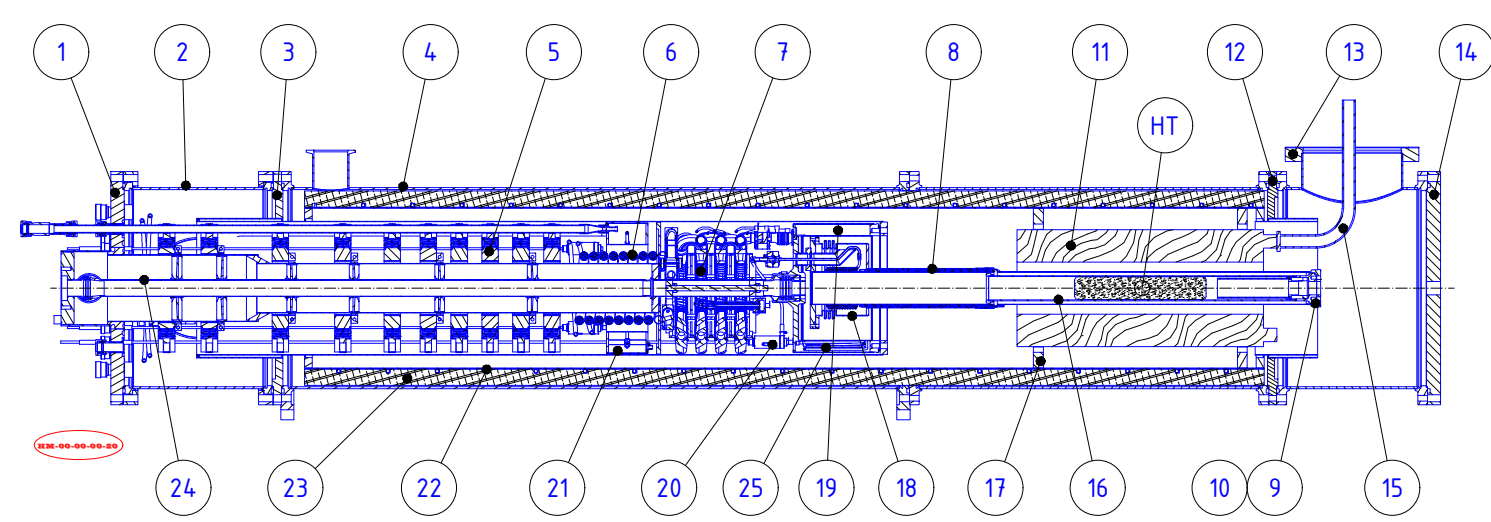

Figure 4: Schematic of the Hydro-Møller atomic trap. 1 - port flange , 2 - cross, 3 - connector flange cryostat, 4 - housing, 5 - high temperature HX, 6 - intermediate temperature HX , 7 - low temperature HX, 8 - final HX, 9 - one-sided film burner, 10 - double-sided film burner, 11 - super conducting solenoid, 12 connector flange, 13 - tees, 14 - output flange, 15 - He4 - connections, 16 - mixing chamber, 17 - thermally insulated mounting, 18 - still, 19 - evaporator with 25-condenser, 20 - needle valves, 21 - separator, 22 - 77 $\mathrm{K}$ shield, 23 - multi layer insulation, 24 - evaporator pumping line

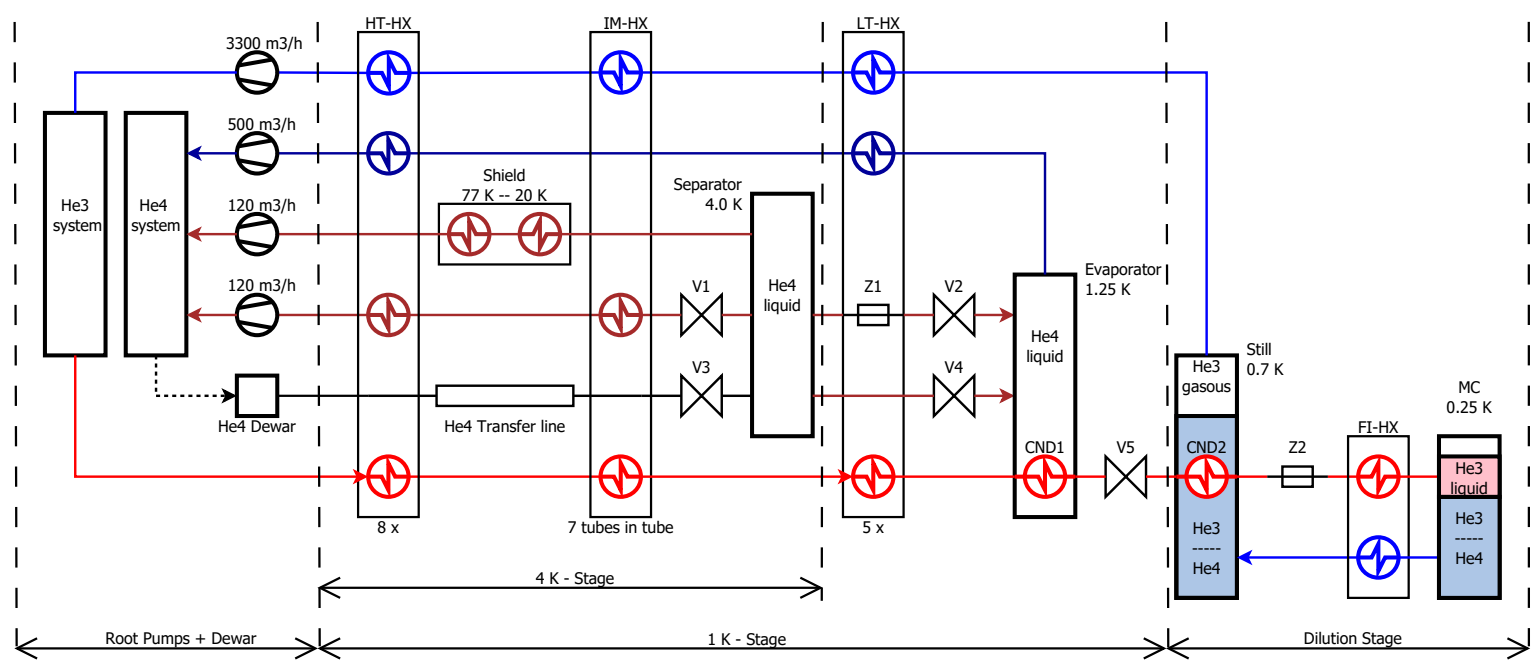

Figure 5: Refrigerator flow chart. HX=Heat exchanger. HT/IM/LT = high, medium and low temperature level of precooling circuit. 


\subsection{Status of component fabrication}

Figures 6 shows the cryostat insert (evaparator pumping line in figure 4) which comprises the beam pipe. It will also carry on its outer cylindrical surface the heat exchangers for the precooling system, one of them is shown in fig. 7. We want to test the precooling system in 2020. The low temperature part containing, among others, the dilution stage will be attached to this support at a later time.

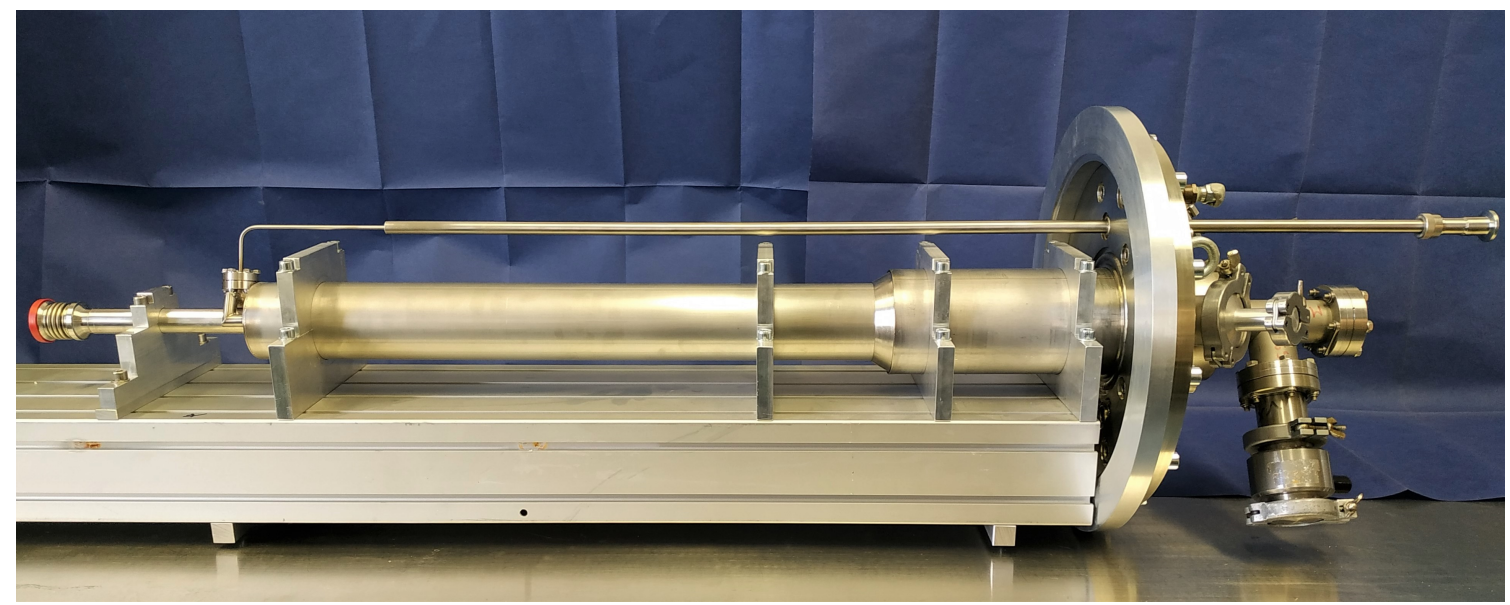

Figure 6: Evaporator pumping line, completely welded using plasma beam, tested at low temperature. Length of device is $\approx 1 \mathrm{~m}$

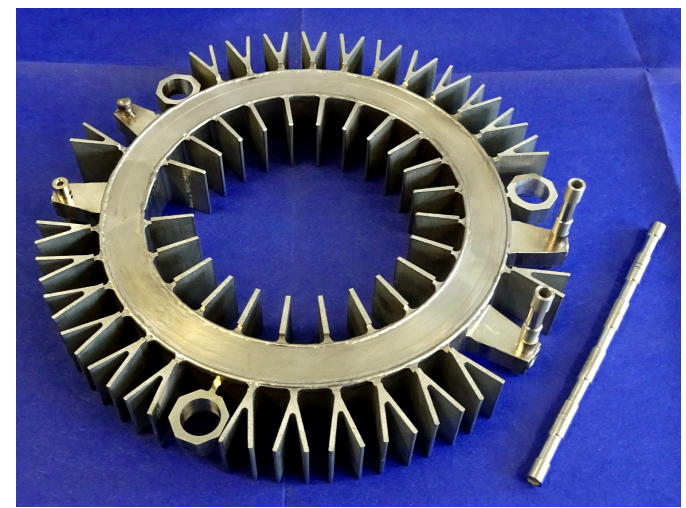

Figure 7: Welded large area stainless steel four ways heat exchanger, with sample connections to weld several such units together. Diameter $\approx 200 \mathrm{~mm}$.

\section{Recent Developments}

\subsection{Installation}

In 2019 several parts of the building in the nuclear physics institute which where formerly not usable due to unclear fire safety issues became available for MESA. This has lead to a revised 
layout of MESA which is shown in fig. 1. The new setting is favorable for the Hydro-Møller since much more space - in particular in the vertical direction - becomes available. The design of the detection system for Møller scattering will also benefit from the new situation.

\section{$3.2{ }^{3} \mathrm{He} /{ }^{4} \mathrm{He}$ dilution stage}

We are negotiating a possible fabrication of these particular challenging parts with the group of Y. Usov from JINR Dubna. A detailed design will be made in 2020 and the parts will become available in 2021.

\subsection{Hydrogen injection}

Two options exist to avoid the freezing out of unwanted spin-species in the system. The first one is to inject only the useful species by a polarized atomic beam source. Sources of polarized hydrogen atoms are well known and have been used in nuclear physics experiments for a long time [12]. Their disadvantages are the relatively high additional complexity and price plus the need to transfer the atoms from high to low temperature without losses. We are presently discussing an alternative, which is the cold hydrogen dissociator [13]. Figure 3.3 illustrates the small size of such a dissociator which makes it possible to integrate it into our cryostat. Because it operates at $1 \mathrm{~K}$ it avoids the problem of clogging. Such dissociators have operated reliably over periods of several months [13] and can deliver sufficient flux to operate the trap.

\subsection{Warm bore solenoid}

The initial design of our cryostat (figure 4) was restricted by vertical headroom of only about $500 \mathrm{~mm}$. This has changed in the new accelerator arrangement where the height is several meters. This allows using a commercial solenoid with vertical cryostat that can comprise the dilution cryostat with the target in its warm bore. This makes the apparatus less complex and maintenance much simpler.

\section{Outlook}

The P2 experiment is expected to start in 2023 and consequently the polarimeter should be commissioned at the same time. Parts of the cryostat have already been fabricated and the dilution stage and the cold hydrogen dissociator can be finalized during 2021. Conventional parts such as the pumping systems, liquid helium distribution can also be purchased until 2022 when testing of the Hydro-Møller could begin.

\section{Acknowledgments}

We want to give special thanks Prof. dr. J.T.M. Walraven UvA, Dr. S.Vasiliev UTU, Dr. T. Niinikoski CERN for valuable informations and discussions. Next thanks head of LT-LNP JINR Dr. Yu. Usov and all staff. 


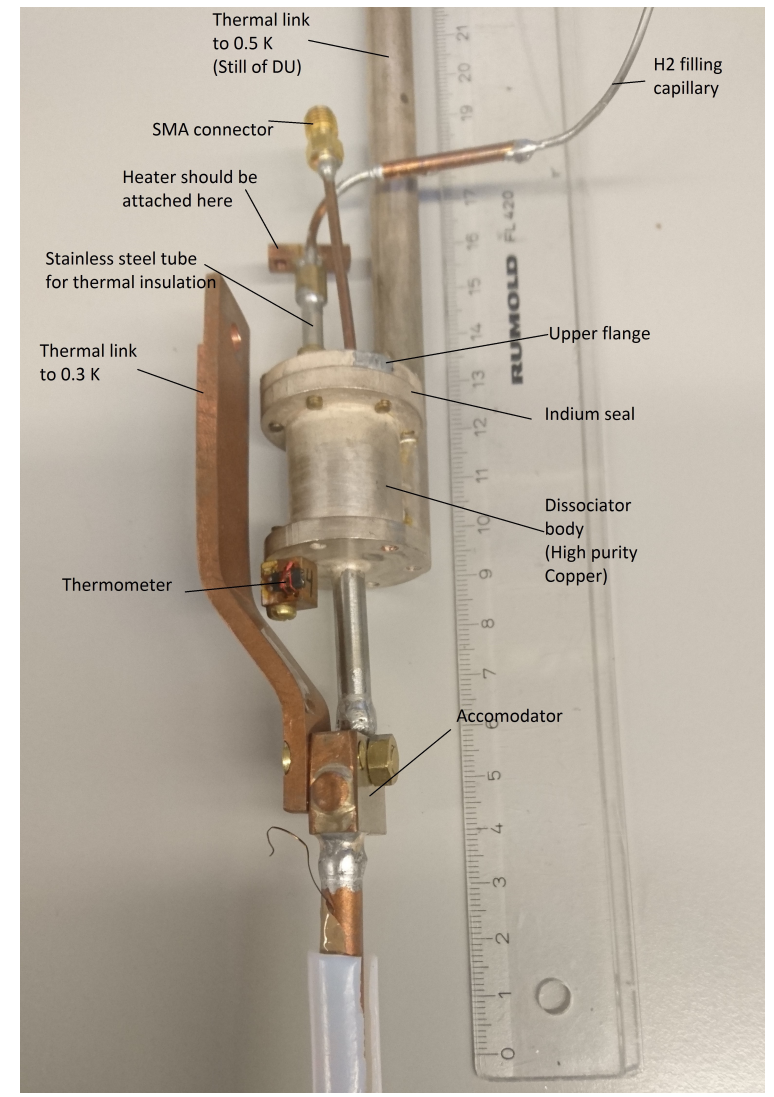

Figure 8: 1K-Dissociator, Thanks to Dr. S. Vasiliev, UTU, Finland

\section{References}

[1] F. Hug et al., MESA - an ERL Project for Particle Physics Experiments, in Proceedings of LINAC2016, East Lansing, MI, US, pp. 313-315, www.jacow.org, (2016).

[2] D. Becker, R. Bucoveanu, C. Grzesik, K. Imai, R. Kempf, M. Molitor et al., The p2 experiment, The European Physical Journal A 54 (2018) 208.

[3] T. Maruyama et al., Systematic study of polarized electron emission from strained GaAs/GaAsP superlattice photocathodes, Appl. Phys. Lett. 85,(13) (2004) 2640.

[4] K. Aulenbacher et al., The polarimetry chain for the P2 experiment, Il nuovo cimeto 32 (2012) 186.

[5] A. Gellrich and J. Kessler, Precision measurement of the sherman asymmetry function for electron scattering from gold, Phys. Rev. A 43 (1991) 204.

[6] SISSA, Proceedings, 17th International Workshop on Polarized Sources, Targets, and Polarimetry (PSTP2017), vol. PSTP2017, SISSA, 2018.

[7] V. Tioukine et al., A Mott polarimeter operating at MeV electron beam energies, REVIEW OF SCIENTIFIC INSTRUMENTS 82 (2011) 033303.

[8] E. Chudakov and V. Luppov, Moller polarimetry with atomic hydrogen targets., Nuclear Science, IEEE Transactions on $\mathbf{5 1}$ (2004) 1533. 
[9] K. Aulenbacher, E. Chudakov, D. Gaskell, J. Grames and K. Paschke, Precision electron beam polarimetry for next generation nuclear physics experiments, International Journal of Modern Physics E 27,7 (2018) 1830004.

[10] L. G. Levchuk, The intra-atomic motion of bound electrons as a possible source of the systematic error in electron-beam polarization measurements by means of a moller polarimeter, NIM A 345 (1994) 496.

[11] J. Walraven and T. Hijmans, Atomic hydrogen in magnetostatic traps, Physica B: Condensed Matter 197 (1994) 417 .

[12] M. Mikirtychyants, R. Engels, K. Grigoryev, H. Kleines, P. Kravtsov, S. Lorenz et al., The polarized $h$ and d atomic beam source for anke at cosy-jülich, Nuclear Instruments and Methods in Physics Research Section A: Accelerators, Spectrometers, Detectors and Associated Equipment 721 (2013) 83

[13] J. Helffrich, M. Maley, M. Krusius and J. C. Wheatley, Hydrogen dissociation below 1 k, Journal of Low Temperature Physics 66 (1987) 277. 